(C) 2006 The Japan Society of Applied Physics

\title{
Implementation of Practical Super-Resolution Near-Field Structure System Using Commercial Drive
}

\author{
Jen Wu Fang, Chia Che Wu*, Allen Liao, Wei Chih Lin and Din Ping Tsai ${ }^{1,2}$ \\ Advanced Research Division, LITE-ON IT Corporation, 5F, 12, Li-Hsin Road, Science-Based Industrial Park, \\ Hsinchu, Taiwan 30078, Republic of China \\ ${ }^{1}$ Department of Physics, National Taiwan University, Taipei, Taiwan 10617, Republic of China \\ ${ }^{2}$ Center for Nanostorage Research, National Taiwan University, Taipei, Taiwan 10617, Republic of China
}

(Received August 12, 2005; accepted December 1, 2005; published online February 24, 2006)

The implementation of a practical super-resolution near-field structure (Super-RENS) system using an existing commercial drive is achieved. Using ZnO-type Super-RENS discs as storage media, the commercial drive can perform mark position recording at a mark size of $220 \mathrm{~nm}$ and correctly retrieve the recorded signal. [DOI: 10.1143/JJAP.45.1383]

KEYWORDS: Super-RENS, ZnO, mark position recording, commercial drive

\section{Introduction}

Recently, a super-resolution near-field structure (SuperRENS) has become one of the most promising technologies toward high-density optical storage. Several generations of mask layers from $\mathrm{Sb}, \mathrm{AgO}_{x}$ to $\mathrm{PtO}_{x}$ were innovated to enhance the signal characteristics and readout stability. ${ }^{1-3)} \mathrm{A}$ ZnO-type Super-RENS disc was also proposed to improve the commercializing practicability and readout stability. ${ }^{4,5)}$ Furthermore, some studies, such as those involving mark position and mark edge recording for a $\mathrm{PtO}_{x}$ structure, were performed to achieve practical optical storage. ${ }^{6,7)}$ However, most studies were carried out using an optical disc tester, whose optical head has nearly excellent optical characteristics. This tester resulted in not only better writing results of the same pattern but also a better readout resolution than a commercial drive. The compatibility of Super-RENS with the commercial drive has not been confirmed. In this study, a commercial drive together with $\mathrm{ZnO}$-type disc is adopted to perform mark position recording to investigate the feasibility of Super-RENS on a commercial drive.

\section{Experimental}

To verify the compatibility of Super-RENS discs with an existing digital versatile disk (DVD) system, an experimental scheme, as shown in Fig. 1, was set up to investigate its feasibility. A LITEON $8 \times \mathrm{DVD} \pm \mathrm{RW}$ drive, which has a laser wavelength of $658 \mathrm{~nm}$ and a numerical aperture (NA) of 0.67 , was equipped with a servo system to write the Super-RENS disc and retrieve the recorded pattern. The servo system was substituted for the servo system of the original drive to obtain a larger flexibility.

The experimental sample discs were fabricated by sputtering. Figure 2 shows that the disc structure is arranged as follows: substrate/dielectric layer/ZnO/dielectric layer/ phase-change layer/protective layer. The dielectric and phase-change layers we adopted are $\mathrm{ZnS}-\mathrm{SiO}_{2}$ and $\mathrm{Ge}_{2} \mathrm{Sb}_{2} \mathrm{Te}_{5}$, respectively. The composition ratio of the dielectric layer $\mathrm{ZnS}-\mathrm{SiO}_{2}$ is $\mathrm{ZnS} 80 \%$ and $\mathrm{SiO}_{2} 20 \%$. A $\mathrm{ZnO}$ thin film functioning as an active layer was fabricated by reactive RF sputtering. The characteristics of the sample disc with a significant readout signal measured beyond the

*E-mail address: clayton_wu@liteonit.com

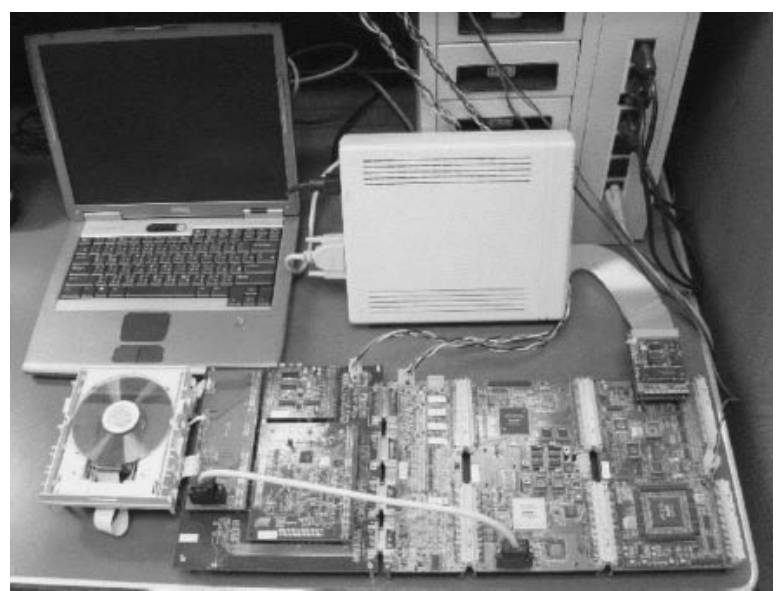

Fig. 1. Photograph of experimental setup.

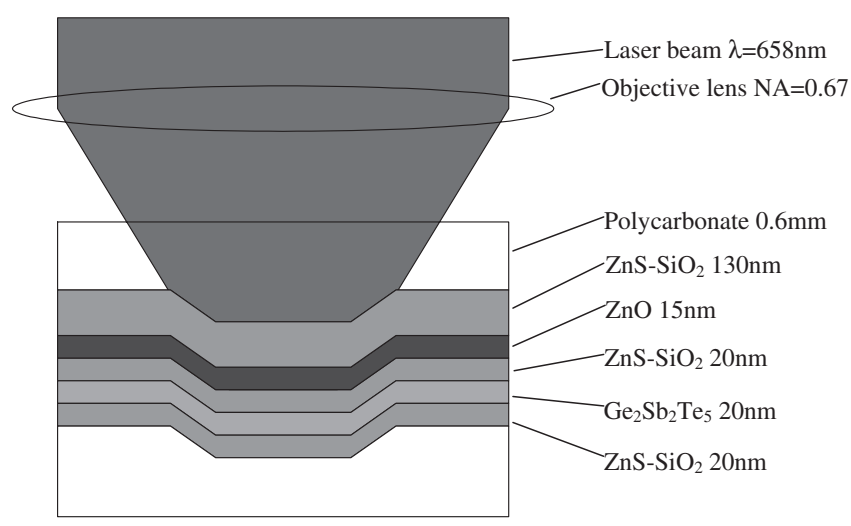

Fig. 2. Structure of sample disc with $\mathrm{ZnO}$ active film.

resolution limit and an excellent readout stability were previously experimentally confirmed. ${ }^{4)}$

\section{Results and Discussion}

To evaluate the readout capability of the commercial drive for the Super-RENS disc, an experiment about carrier-tonoise ratio (CNR) at various recorded patterns from $110 \mathrm{~nm}$ to $500 \mathrm{~nm}$ was first carried out. Marks were recorded with a $50 \%$ duty cycle of writing pulse, and the writing and bottom powers were 17 and $0.7 \mathrm{~mW}$, respectively. The sample disc 


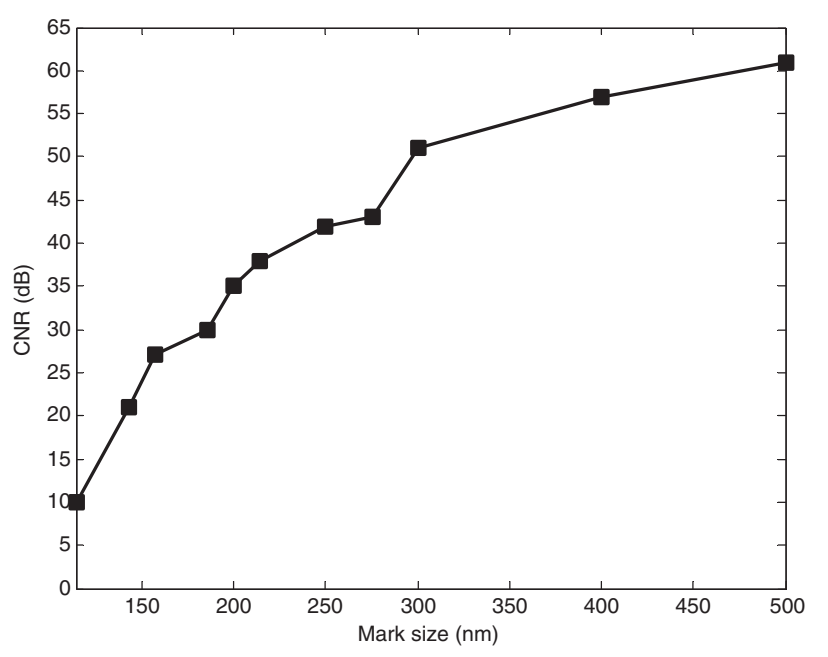

Fig. 3. CNRs at various recorded mark sizes.

was recorded at a constant linear velocity (CLV) of $3.5 \mathrm{~m} / \mathrm{s}$. The readout signals of the recorded marks were measured at the same constant linear velocity and the read power was $5 \mathrm{~mW}$. Figure 3 illustrates that even at the diffraction limit $(\sim 270 \mathrm{~nm})$, the commercial drive can retrieve a significantly high CNR. For a mark size of $200 \mathrm{~nm}$, the CNR is around $35 \mathrm{~dB}$. If the duty cycle of writing pulse is changed to $40 \%$, CNR can be enhanced to around $38 \mathrm{~dB}$.

Mark position recording and readout were adopted to verify the feasibility of implementing the Super-RENS system with a commercial drive. Because the CNR of a $250 \mathrm{~nm}$ mark was $42 \mathrm{~dB}, 250 \mathrm{~nm}$ was selected to be the mark length of mark position recording. The recording pattern had $250 \mathrm{~nm}$ mark trains placed in between $600 \mathrm{~nm}$ spaces with a number of mark trains from one to six. The readout experimental results obtained using a conventional equalizer are shown in Fig. 4. Figure 4(a) reveals that when the readout power, $1.3 \mathrm{~mW}$, is less than the threshold power required for inducing the super-resolution effect, the CNR of the $250 \mathrm{~nm}$ marks is almost $0 \mathrm{~dB}$. Thus, the $250 \mathrm{~nm}$ marks cannot be detected. Figure 4(b) shows that when the readout power, $4 \mathrm{~mW}$, is larger than the threshold power required for inducing the super-resolution effect, the CNR of the $250 \mathrm{~nm}$ marks is $42 \mathrm{~dB}$. Thus, the isolated mark and mark trains recorded on the Super-RENS disc can be clearly retrieved. Moreover, mark position recording with a mark length of $220 \mathrm{~nm}$ was also achieved as shown in Fig. 5. The CNR of $220 \mathrm{~nm}$ was $38 \mathrm{~dB}$ as the read power was $4 \mathrm{~mW}$. Although each first bit of the mark trains was slightly diminished, the pattern could still be distinguished with the read power of $4 \mathrm{~mW}$. Consequently, the experimental results validate that the Super-RENS discs can be preliminarily compatible with the existing commercial drive.

\section{Conclusions}

A commercial drive was verified to be capable of recording a fixed pattern and retrieving the recorded signal for a ZnO type Super-RENS disc. Furthermore, mark position recordings at mark sizes of 250 and $220 \mathrm{~nm}$ mark size were also successfully retrieved, confirming the feasibility of using the existing commercial drive as the SuperRENS drive only by modifying firmware.

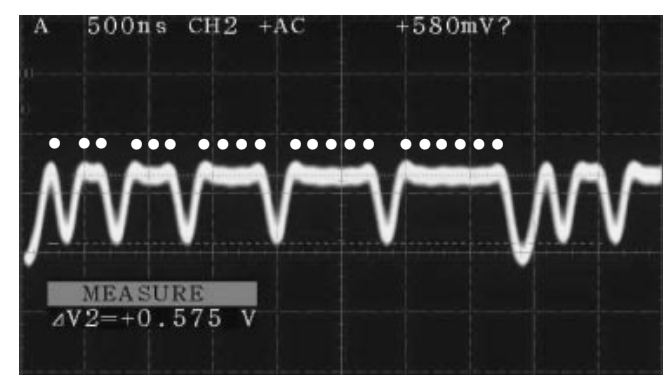

(a)

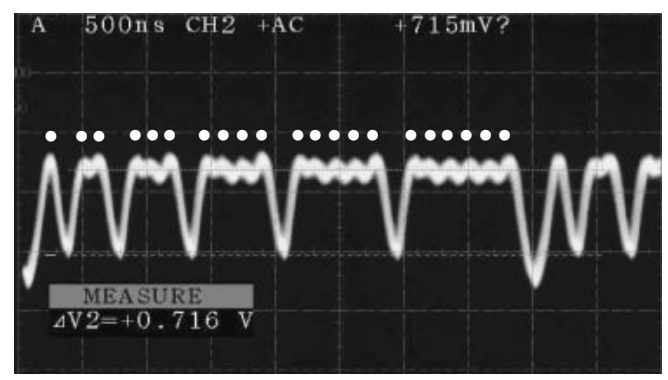

(b)

Fig. 4. Readout signals retrieved from recorded pattern of $250 \mathrm{~nm}$ mark length with different read powers: (a) $1.3 \mathrm{~mW}$ and (b) $4 \mathrm{~mW}$.

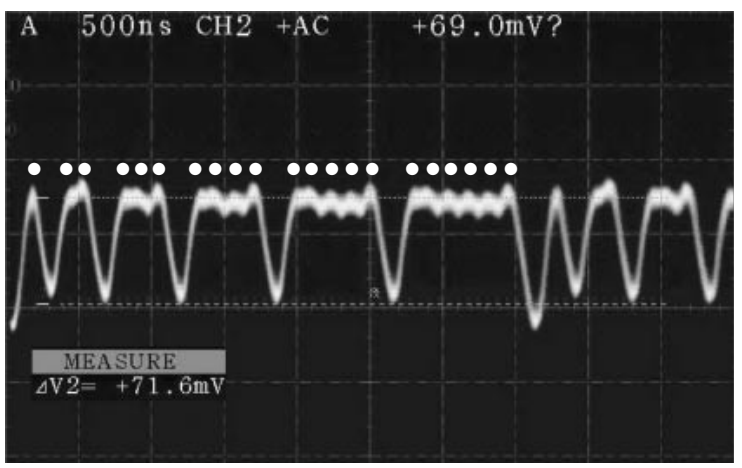

Fig. 5. Readout signals retrieved from recorded pattern of $220 \mathrm{~nm}$ mark length.

\section{Acknowledgement}

The authors are grateful for the research support from the Ritek Corporation.

1) J. Tominaga, T. Nakano and N. Atoda: Appl. Phys. Lett. 73 (1998) 2078.

2) H. Fuji, J. Tominaga, L. Men, T. Nakano, H. Katayama and N. Atoda: Jpn. J. Appl. Phys. 39 (2000) 980.

3) T. Kikukawa, H. Nakano, T. Shima and J. Tominaga: Appl. Phys. Lett. 81 (2002) 4697.

4) W. C. Lin, T. S. Kao, H. H. Chang, Y. H. Lin, Y. H. Fu, C. T. Wu, K. H. Chen and D. P. Tsai: Jpn. J. Appl. Phys. 42 (2003) 1029.

5) M. Yamamoto, G. Mori, H. Tajima, N. Takamori and A. Takahashi: Jpn. J. Appl. Phys. 43 (2004) 4959.

6) H. Fuji, T. Kikukawa and J. Tominaga: Jpn. J. Appl. Phys. 42 (2003) L589.

7) H. Fuji, T. Kikukawa and J. Tominaga: Jpn. J. Appl. Phys. 43 (2004) 4212. 